\begin{tabular}{|c|l|}
\hline Title & $\begin{array}{l}\text { Existence and structure of infinitely degenerate zero-energy ground states of a W ess Zumino type model in } \\
\text { supersymmetric quantum mechanics }\end{array}$ \\
\hline Author(s) & Ogurisu, O. \\
\hline Citation & Hokkaido University Preprint Series in Mathematics, 179, 1-26 \\
\hline Issue Date & 1993-01 \\
\hline DOI & 10.14943/83323 \\
\hline Doc URL & http://hdl.handle.net/2115/68925 \\
\hline Type & bulletin (article) \\
\hline File Information & pre179.pdf \\
\hline
\end{tabular}

Instructions for use 
EXISTENCE AND STRUCTURE OF INFINITELY DEGENERATE ZEROENERGY GROUND STATES OF A WESS-ZUMINO TYPE MODEL IN SUPERSYMMETRIC QUANTUM MECHANICS

Osamu Ogurisu

Series $\sharp 179$. January 1993 


\section{HOKKAIDO UNIVERSITY \\ PREPRINT SERIES IN MATHEMATICS}

\#151: G. Ishikawa, Developable of a curve and determinacy relative to osculation-type, 15 pages. 1992.

$\sharp 152$ : H. Kubo, Global existence of solutions of semilinear wave equations with data of non compact support in odd space dimensions, 25 pages. 1992.

\#153: Y. Watatani, Lattices of intermediate subfactors, 33 pages. 1992.

\#154: T. Ozawa, On critical cases of Sobolev inequalities, 11 pages. 1992.

$\sharp 155$ : M. Ohnuma, M. Sato, Singular degenerate parabolic equations with applications to geometric evolutions, 20 pages. 1992.

$\sharp 156$ : S. Izumiya, Perestroikas of optical wave fronts and graphlike Legendrian unfoldings, 13 pages. 1992.

$\sharp 157$ : A. Arai, Momentum operators with gauge potentials, local quantization of magnetic flux, and representation of canonical commutation relations, 11 pages. 1992.

$\sharp 158$ : S. Izumiya, W.L. Marar, The Euler number of a topologically stable singular surface in a 3-manifold, 11 pages. 1992.

\#159: T. Hibi, Cohen-Macaulay types of Cohen-Macaulay complexes, 26 pages. 1992.

$\sharp 160$ : A. Arai, Properties of the Dirac-Weyl operator with a strongly singular gauge potential, 26 pages. 1992.

$\sharp 161$ : A. Arai, Dirac operators in Boson-Fermion Fock spaces and supersymmetric quantum field theory, 30 pages. 1992.

$\sharp 162$ : S. Albeverio, K. Iwata, T. Kolsrud, Random parallel transport on surfaces of finite type, and relations to homotopy, 8 pages. 1992.

$\#$ 163: S. Albeverio, K. Iwata, T. Kolsrud, Moments of random fields over a family of elliptic curves, and modular forms, 9 pages. 1992.

$\sharp 164: \quad$ Y. Giga, M. Sato, Neumann problem for singular degenerate parabolic equations, 12 pages. 1992.

\# 165: J. Wierzbicki, Y. Watatani, Commuting squares and relative entropy for two subfactors, 18 pages. 1992.

$\sharp 166$ : Y. Okabe, A new algorithm driven from the view-point of the fluctuation-dissipation theorem in the theory of $\mathrm{KM}_{2} \mathrm{O}$-Langevin equations, 13 pages. 1992.

$\sharp 167$ : Y. Okabe, H. Mano and Y. Itoh, Random collision model for interacting populations of two species and its strong law of large numbers, 14 pages. 1992.

$\sharp 168:$ A. Inoue, On the equations of stationary precesses with divergent diffusion coefficients, 25 pages. 1992.

$\sharp 169: \quad$ T. Ozawa, Remarks on quadratic nonlinear Schrödinger equations, 19 pages. 1992.

$\sharp 170$ : T. Fukui, Y. Giga, Motion of a graph by nonsmooth weighted curvature, 11 pages. 1992.

$\sharp 171$ : J. Inoue, T. Nakazi, Finite dimensional solution sets of extremal problems in $\mathrm{H}^{1}, 10$ pages. 1992.

$\sharp 172$ : S. Izumiya, A characterization of complete integrability for partial differential equations of first order, 6 pages. 1992.

$\sharp 173$ : T. Suwa, Unfoldings of codimension one complex analytic foliation singularities, 49 pages. 1992.

$\sharp 174$ : T. Ozawa, Wave propagation in even dimensional spaces, 15 pages. 1992.

$\sharp 175: \quad$ S. Izumiya, Systems of Clairant type, 7 pages. 1992.

$\sharp 176$ : A. Hoshiga, The initial value problems for quasi-linear wave equations in two space dimensions with small data, 25 pages. 1992.

\#177: K. Sugano, On bicommutators of modules over H-separable extension rings III, 9 pages. 1993.

$\sharp 178$ : T. Nakazi, Toeplitz operators and weighted norm inequalities, 17 pages. 1993. 


\title{
EXISTENCE AND STRUCTURE OF INFINITELY DEGENERATE ZERO-ENERGY GROUND STATES OF A WESS-ZUMINO TYPE MODEL IN SUPERSYMMETRIC QUANTUM MECHANICS
}

\author{
OSAMU OGURISU \\ Department of Mathematics, Hokkaido University \\ Sapporo 060, Japan \\ 1, October 1992
}

\begin{abstract}
It is known that the $\mathrm{N}=2$ Wess-Zumino supersymmetric quantum mechanical model with the superpotential $V(z)=\lambda e^{\alpha z}(\lambda \in \mathbb{C} \backslash\{0\}, \alpha>0)$ has infinitely many bosonic zeroenergy ground states and no fermionic zero-energy ground states [A. Arai, J. Math. Phys. 30 (1989), 1164]. In this paper, we extend these results to a more general model. The main results include the following: (1) identification of the spectra of the Hamiltonian $H$ of the model; (2) non-Fredholmness of a supercharge of the model, which is a Dirac type operator; (3) existence of infinitely many bosonic zero-energy states of $H$; (4) non-existence of fermionic zero-energy states of $H$.
\end{abstract}

PACS: 02.30.Jr, 02.30.Tb, 03.65.Db, 03.65.Ge

to appear in Journal of Mathematical Physics 


\section{INTRODUCTION}

It is known that the $\mathrm{N}=2$ Wess-Zumino (WZ) model in supersymmetric quantum mechanics, ${ }^{1,2}$ which describes the interaction between a complex bosonic degree $z \in \mathbb{C}$ and two fermionic degrees of freedom, has degenerate zero-energy ground states for some classes of superpotentials $V(z) \cdot{ }^{3-6}$ The following (V1)-(V3) hold:

(V1) If $V(z)$ is a polynomial of $z$, then the number of the bosonic zero-energy ground states $n_{b}$ is equal to $\operatorname{deg} V-1 .^{3,4}$

(V2) If $V(z)=\lambda z^{-p}(\lambda \in \mathbb{C} \backslash\{0\}, p \in \mathbb{N})$, then $n_{b}=p-1 .^{5}$;

(V3) If $V(z)=\lambda e^{\alpha z}(\lambda \in \mathbb{C} \backslash\{0\}, \alpha>0)$, then $n_{b}=\infty .^{6}$

In all the cases, the number of the fermionic zero-energy states is equal to 0 ("vanishing theorem"). ${ }^{3-6}$

In the case where $V(z)=\lambda z^{p}(\lambda \in \mathbb{C} \backslash\{0\}, p \in \mathbb{N})$, a special case of (V1), and in the case (V2), the mathematical structure of the degenerate ground states has been analyzed in detail. ${ }^{4,5}$ Both of these cases have some common structures:

(i) There exists a strongly continuous one parameter unitary group acting as a symmetry group in the quantum system governed by the bosonic part $H_{+}$of the Hamiltonian $H$ of the model.

(ii) The generator $L$ of the symmetry group has purely discrete spectrum and infinitely many eigenspaces, each of which reduces $H_{+}$.

(iii) There exist some eigenspaces of $L$ such that $H_{+}$has a unique zero-energy state on each of them. (Hence the number of such eigenspaces is equal to the number of the bosonic zero-energy states.)

(iv) $H_{+}$has generalized zero-energy states on each of the eigenspaces of $L$.

However, in the case (V3), it has not been known whether the ground states have a structure similar to the cases (V1) and (V2). In this paper we present a new class of superpotentials containing the case (V3) such that each superpotential in the class defines a WZ type model with the following structures:

(i') There exists a symmetry group of the bosonic Hamiltonian $H_{+}$.

(ii') The generator $N_{+}$of the symmetry group has no point spectrum and every $p \in \mathbb{R}$ is a generalized eigenvalue of $N_{+}$. Each generalized eigenspace $\mathcal{H}_{p}$ of $N_{+}$is regarded as 
a Hilbert space and "reduces" $H_{+}$.

(iii') There exists an interval $\mathrm{I}$ in $\mathbb{R}$ such that for every $p \in \mathrm{I}, H_{+}$has a unique zero-energy state on $\mathcal{H}_{p}$.

(iv') There exist infinitely many zero-energy ground states of $H_{+}$.

(v') There exist infinitely many generalized zero-energy states of $H_{+}$.

For another class of superpotentials containing the case (V3), we prove in a way similar to the case of $H_{+}$the vanishing theorem and that there exist infinitely many generalized fermionic zero-energy states.

We also prove that the spectrum of $H_{ \pm}$is equal to $[0, \infty)$, which implies that a supercharge of the model is not semi-Fredholm. This suggests the instability of ker $H_{-}$. (See Remark 3.7)

The plan of this paper is as follows. In Sec. II, we introduce a class of superpotentials containing $\lambda e^{\alpha z}$ and define the WZ type model with a superpotential in the class. In Sec. III, we identify the spectra of the Hamiltonian of the model. In Sec. IV, we consider conditions for the superpotential under which there exist infinitely many zero-energy ground states of $H_{+}$, and give a concrete class of superpotentials which satisfy these conditions. In Sec.V, we consider conditions for the superpotential under which there exists no fermionic zeroenergy ground states. In Appendix we summarize a few known results in a theory of ordinary differential equation, which are used in the main text. 


\section{Models With SUPERPotentials Similar to $\exp (\alpha z)$}

In this section we define a class of functions on $\mathbb{C}$ and discuss the $\mathrm{N}=2 \mathrm{WZ}$ type model with a superpotential $V$ in the class. We identify $z \in \mathbb{C}$ with $x+i y$ where $x, y \in \mathbb{R}$. We denote $\partial / \partial z$ and $\partial / \partial \bar{z}$ by $\partial$ and $\bar{\partial}$, respectively.

Let $\alpha$ be an arbitrary positive constant.

Definition 2.1. A function $V: \mathbb{C} \rightarrow \mathbb{C}$ is said to be in $\mathcal{V}_{0}$ if there exists $v \in C^{\infty}(\mathbb{R})$ such that

$$
\partial V(z)=v(x) e^{i \alpha y} .
$$

The Hilbert space of state vectors of the $\mathrm{N}=2 \mathrm{WZ}$ type model with a superpotential $V \in \mathcal{V}_{0}$ is realized as

$$
\mathcal{H}=L^{2}\left(\mathbb{C} ; \mathbb{C}^{4}\right)=L^{2}\left(\mathbb{R}^{2} ; \mathbb{C}^{4}\right)=\mathcal{H}_{+} \oplus \mathcal{H}_{-}
$$

where

$$
\mathcal{H}_{+}=\left\{\left(\begin{array}{l}
f \\
g
\end{array}\right) \mid f, g \in L^{2}(\mathbb{C})\right\}=L^{2}\left(\mathbb{C} ; \mathbb{C}^{2}\right)
$$

and

$$
\mathcal{H}_{-}=\left\{\left(\begin{array}{l}
f \\
g
\end{array}\right) \mid f, g \in L^{2}(\mathbb{C})\right\}=L^{2}\left(\mathbb{C} ; \mathbb{C}^{2}\right)
$$

are the subspaces of "bosonic" and "fermionic" states, respectively.

Let $V \in \mathcal{V}_{0}$. One of the supercharges of the model with the superpotential $V$ is given by

$$
Q=\left(\begin{array}{cc}
0 & Q_{-} \\
Q_{+} & 0
\end{array}\right)
$$

acting in $\mathcal{H}$, where $Q_{-}$and $Q_{+}$are operators defined by

$$
Q_{-}=\left(\begin{array}{cc}
i \partial V & -\partial \\
-\bar{\partial} & -i(\partial V)^{*}
\end{array}\right) \text { in } \mathcal{H}_{-}
$$

and

$$
Q_{+}=\left(\begin{array}{cc}
-i(\partial V)^{*} & \partial \\
\bar{\partial} & i \partial V
\end{array}\right) \text { in } \mathcal{H}_{+} .
$$

We put $D=C_{0}^{\infty}\left(\mathbb{R}^{2} ; \mathbb{C}^{4}\right)$. In the same way as in the proof of Proposition 2.1 in Ref. 6 , we can prove the following proposition, because the holomorphic property of $V(z)$ is not used in the proof there. 
Proposition 2.2. The operator $Q$ is essentially self-adjoint on $D$. Further, every power of $Q$ is essentially self-adjoint on $D$.

We denote the closure of $Q$ by the same symbol $Q$ and define the non-negative self-adjoint operator $H$ by

$$
H=Q^{2}=\left(\begin{array}{cc}
H_{+} & 0 \\
0 & H_{-}
\end{array}\right)
$$

with

$$
H_{+}=Q_{+}^{*} Q_{+}, \quad H_{-}=Q_{+} Q_{+}^{*}
$$

The operator $H$ is the Hamiltonian of the model, called the supersymmetric Hamiltonian. We call $H_{+}$(resp. $H_{-}$) the bosonic (resp. fermionic) Hamiltonian. We have

$$
H_{+}=H_{0}+\left(\begin{array}{cc}
0 & -i \partial^{2} V \\
i\left(\partial^{2} V\right)^{*} & 0
\end{array}\right)
$$

and

$$
H_{-}=H_{0}+\left(\begin{array}{cc}
0 & -i(\bar{\partial} \partial V)^{*} \\
i \bar{\partial} \partial V & 0
\end{array}\right)
$$

on $D$, where

$$
H_{0}=\left(-\partial \bar{\partial}+|\partial V|^{2}\right)\left(\begin{array}{ll}
1 & 0 \\
0 & 1
\end{array}\right)
$$




\section{Spectra of the Hamiltonians}

We denote by $\sigma(A), \sigma_{p}(A)$ and $D(A)$ the spectrum, the point spectrum and the domain of an operator $A$, respectively.

In this section we identify the spectra of the Hamiltonian of the models with potentials in a subclass of $\mathcal{V}_{0}$. Moreover, we show that the Hamiltonians $H_{ \pm}$and the supercharge $Q$ are not semi-Fredholm.

We first prove these facts in the case where $\partial V(z)=\lambda e^{\alpha z}$. In this case we can identify the point spectrum of $H_{-}$, too.

Theorem 3.1. Let $\partial V(z)=\lambda e^{\alpha z}$ with $\lambda \in \mathbb{C} \backslash\{0\}$. Then

$$
\sigma\left(H_{ \pm}\right)=[0, \infty), \quad \sigma_{p}\left(H_{-}\right)=\emptyset
$$

Proof. In the present case, we have

$$
H_{-}=-\partial \bar{\partial}+|\partial V|^{2}=\left(\begin{array}{cc}
K & 0 \\
0 & K
\end{array}\right),
$$

where

$$
K=-\frac{1}{4} \frac{\partial^{2}}{\partial x^{2}}-\frac{1}{4} \frac{\partial^{2}}{\partial y^{2}}+|\lambda|^{2} e^{2 \alpha x}
$$

It follows that $\sigma\left(H_{-}\right)=\sigma(K), \sigma_{p}\left(H_{-}\right)=\sigma_{p}(K)$. Let $H_{x}$ and $H_{y}$ be the self-adjoint operators in $L^{2}(\mathbb{R})$ defined by

$$
H_{x}=-\frac{1}{4} \frac{d^{2}}{d x^{2}}+|\lambda|^{2} e^{2 \alpha x}, \quad H_{y}=-\frac{1}{4} \frac{d^{2}}{d y^{2}} .
$$

We denote by $I$ the identity on $L^{2}(\mathbb{R})$. Then

$$
K=H_{x} \otimes I+I \otimes H_{y}
$$

as an operator on $L^{2}\left(\mathbb{R}^{2}\right)=L^{2}(\mathbb{R}) \otimes L^{2}(\mathbb{R})$. It is well known that, for self-adjoint operators $A_{1}$ and $A_{2}$,

$$
\begin{aligned}
& \sigma\left(A_{1} \otimes I+I \otimes A_{2}\right)=\overline{\left\{\mu=\mu_{1}+\mu_{2} \mid \mu_{j} \in \sigma\left(A_{j}\right), j=1,2\right\}}, \\
& \sigma_{p}\left(A_{1} \otimes I+I \otimes A_{2}\right)=\overline{\left\{\mu=\mu_{1}+\mu_{2} \mid \mu_{j} \in \sigma_{p}\left(A_{j}\right), j=1,2\right\}}
\end{aligned}
$$

and that $\sigma\left(H_{y}\right)=[0, \infty)$ and $\sigma_{p}\left(H_{y}\right)=\emptyset$. Hence we see that $\sigma_{p}(K)=\emptyset$. Thus, $\sigma_{p}\left(H_{-}\right)=\emptyset$. We next prove that $\sigma\left(H_{x}\right) \supset[0, \infty)$. 
We use the following basic criterion: Let $A$ be a self-adjoint operator. Then $\nu \in \sigma(A)$ if and only if there exist a sequence $\left\{\phi_{n}\right\}_{n \in \mathbb{N}} \subset D(A)$ such that $\left\|\phi_{n}\right\|=1$ and $\left\|(A-\nu) \phi_{n}\right\| \rightarrow 0$ as $n \rightarrow \infty$. Let $\mu \geq 0$. We prove that $\mu^{2} \in \sigma\left(H_{x}\right)$. Let $c=\sqrt{\pi}$ and for each $n \in \mathbb{N}$

$$
\phi_{n}(x)=\frac{1}{\sqrt{n c}} e^{2 i \mu x} e^{-x^{2} / 2 n^{2}} .
$$

Then $\phi_{n} \in D\left(d^{2} / d x^{2}\right) \cap D\left(e^{2 \alpha x}\right)$ and $\left\|\phi_{n}\right\|=1$. Let $T_{n}$ be a shift operator on $L^{2}(\mathbb{R})$ defined by

$$
T_{n} f(x)=f\left(x+n^{3}\right)
$$

Then the following hold:

(1) $\left\|T_{n} f\right\|=\|f\|$ for $f \in L^{2}(\mathbb{R})$,

(2) $\left\|e^{2 \alpha x} T_{n} f\right\|=e^{-2 \alpha n^{3}}\left\|e^{2 \alpha x} f\right\| \quad$ for $f \in D\left(e^{2 \alpha x}\right)$,

(3) $T_{n}\left(-\frac{1}{4} \frac{d^{2}}{d x^{2}}-\mu^{2}\right)=\left(-\frac{1}{4} \frac{d^{2}}{d x^{2}}-\mu^{2}\right) T_{n}$ on $D\left(\frac{d^{2}}{d x^{2}}\right)$.

Let $f_{n}=T_{n} \phi_{n}$. Then $\left\|f_{n}\right\|=1$ and $f_{n} \in D\left(d^{2} / d x^{2}\right) \cap D\left(e^{2 \alpha x}\right)$. We have

$$
\left\|\left(H_{x}-\mu^{2}\right) f_{n}\right\| \leq\left\|\left(-\frac{1}{4} \frac{d^{2}}{d x^{2}}-\mu^{2}\right) \phi_{n}\right\|+|\lambda|^{2} e^{-2 \alpha n^{3}}\left\|e^{2 \alpha x} \phi_{n}\right\| .
$$

We have

$$
\left(-\frac{1}{4} \frac{d^{2}}{d x^{2}}-\mu^{2}\right) \phi_{n}=\frac{1}{4 n^{2}}\left(-\frac{x^{2}}{n^{2}}+4 i \mu x+1\right) \phi_{n} .
$$

Hence

$$
\left\|\left(-\frac{1}{4} \frac{d^{2}}{d x^{2}}-\mu^{2}\right) \phi_{n}\right\| \leq \frac{1}{4 n^{2}}\left\{\frac{1}{n^{2}}\left\|x^{2} \phi_{n}\right\|+4 \mu\left\|x \phi_{n}\right\|+\left\|\phi_{n}\right\|\right\} .
$$

We can easily verify that

$$
\left\|x^{2} \phi_{n}\right\|=n^{2} \sqrt{\frac{1}{c} \int_{\mathbb{R}} y^{4} e^{-y^{2}} d y} \text { and }\left\|x \phi_{n}\right\|=n \sqrt{\frac{1}{c} \int_{\mathbb{R}} y^{2} e^{-y^{2}} d y}
$$

for all $n \in \mathbb{N}$. Therefore, the first term on the right hand side of (3.1) converges to 0 as $n \rightarrow \infty$. We have $\left\|e^{2 \alpha x} \phi_{n}\right\|=\exp \left(2 \alpha^{2} n^{2}\right)$. Thus, since $\alpha$ is positive, the second term on the right hand side of (3.1) converges to 0 as $n \rightarrow \infty$. Hence

$$
\left\|\left(H_{x}-\mu^{2}\right) f_{n}\right\| \rightarrow 0 \quad \text { as } n \rightarrow \infty
$$


Hence $\mu^{2} \in \sigma\left(H_{x}\right)$. Therefore we obtain that $[0, \infty) \subset \sigma\left(H_{x}\right)$. Since $H_{x}$ is non-negative selfadjoint, it follows that $\sigma\left(H_{x}\right)=[0, \infty)$, which implies $\sigma(K)=[0, \infty)$. Hence $\sigma\left(H_{-}\right)=[0, \infty)$.

In general, for any densely defined closed linear operator $A$ from a Hilbert space to another one, $\sigma\left(A^{*} A\right) \backslash\{0\}=\sigma\left(A A^{*}\right) \backslash\{0\}$. (See Ref.7) Hence $\sigma\left(H_{+}\right) \backslash\{0\}=\sigma\left(H_{-}\right) \backslash\{0\}$. It follows that $\sigma\left(H_{+}\right)=[0, \infty)$.

Corollary 3.2. Let $\partial V(z)=\lambda e^{\alpha z}$ with $\lambda \in \mathbb{C} \backslash\{0\}$. Then $H_{ \pm}$is not semi-Fredholm.

Proof. It is known that a densely defined closed linear operator $A$ is semi-Fredholm if and only if (1) inf $\sigma\left(A^{*} A\right) \backslash\{0\}>0$ and (2) at least one of $\operatorname{dim} \operatorname{ker} A^{*} A$ and $\operatorname{dim} \operatorname{ker} A A^{*}$ is finite. By Theorem 3.1, it follows that $\sigma\left(H_{ \pm}^{*} H_{ \pm}\right)=\sigma\left(H_{ \pm}^{2}\right)=[0, \infty)$, which implies that $H_{ \pm}$is not semi-Fredholm.

We introduce a subclass $\mathcal{V}_{1}$ of $\mathcal{V}_{0}$

Definition 3.3. We say that $V$ is in $\mathcal{V}_{1}$ if $V \in \mathcal{V}_{0}$ and there exist constants $c$ and $\beta>0$ such that

$$
\left|v^{(j)}(x)\right| \leq c e^{\beta x}, \quad x \in \mathbb{R}, \quad j=0,1
$$

where $v^{(j)}(x)=d^{j} v(x) / d x^{j}$.

For this subclass we obtain the following results.

Theorem 3.4. Suppose $V \in \mathcal{V}_{1}$. Then

$$
\sigma\left(H_{ \pm}\right)=[0, \infty)
$$

Proof. Let

$$
H_{0}=-\frac{1}{4} \frac{d^{2}}{d x^{2}} \otimes I-I \otimes \frac{1}{4} \frac{d^{2}}{d y^{2}}+|v(x)|^{2} \otimes I \quad \text { in } \quad L^{2}\left(\mathbb{R}^{2}\right) .
$$

We can write

$$
H_{-}=\left(\begin{array}{cc}
H_{0} & 0 \\
0 & H_{0}
\end{array}\right)+\frac{i}{2}\left(\begin{array}{cc}
0 & \overline{\left(v^{\prime}-\alpha v\right)} \otimes e^{-i \alpha y} \\
\left(v^{\prime}-\alpha v\right) \otimes e^{i \alpha y} & 0
\end{array}\right) .
$$

Let $\mu \geq 0$. We prove that $2 \mu^{2} \in \sigma\left(H_{-}\right)$. Let $\phi_{n}$ and $f_{n}$ be the functions in the proof of Theorem 3.1. Then,

$$
\left\|\left(-\frac{1}{4} \frac{d^{2}}{d x^{2}}-\mu^{2}\right) \phi_{n}\right\| \rightarrow 0, \quad\left\|\left(-\frac{1}{4} \frac{d^{2}}{d x^{2}}-\mu^{2}\right) f_{n}\right\| \rightarrow 0 \text { as } n \rightarrow \infty .
$$


Moreover,

$$
\begin{aligned}
& \left\||v|^{2} f_{n}\right\| \leq c^{2}\left\|e^{2 \beta x} f_{n}\right\| \rightarrow 0 \text { as } n \rightarrow \infty \\
& \left\|\left(v^{\prime}-\alpha v\right) f_{n}\right\| \text { and }\left\|\overline{\left(v^{\prime}-\alpha v\right)} f_{n}\right\| \leq(c+\alpha c)\left\|e^{\beta x} f_{n}\right\| \rightarrow 0 \text { as } n \rightarrow \infty
\end{aligned}
$$

Let

$$
\Phi_{n}=\frac{1}{\sqrt{2}}\left(\begin{array}{c}
f_{n} \otimes \phi_{n} \\
f_{n} \otimes \phi_{n}
\end{array}\right) .
$$

Then $\left\|\Phi_{n}\right\|=1$. We have

$$
\begin{aligned}
\left\|\left(H_{-}-2 \mu^{2}\right) \Phi_{n}\right\|^{2}= & \left\|\left(H_{0}-2 \mu^{2}-\frac{i}{2} \overline{\left(v^{\prime}-\alpha v\right)} \otimes e^{-i \alpha y}\right) f_{n} \otimes \phi_{n}\right\|^{2} \\
& +\left\|\left(H_{0}-2 \mu^{2}+\frac{i}{2}\left(v^{\prime}-\alpha v\right) \otimes e^{i \alpha y}\right) f_{n} \otimes \phi_{n}\right\|^{2} .
\end{aligned}
$$

We have

$$
\begin{aligned}
& \left\|\left(H_{0}-2 \mu^{2}-\frac{i}{2} \overline{\left(v^{\prime}+\alpha v\right)} \otimes e^{-i \alpha y}\right) f_{n} \otimes \phi_{n}\right\| \\
& \quad \leq\left\|\left(-\frac{1}{4} \frac{d^{2}}{d x^{2}}-\mu^{2}\right) f_{n}\right\|+\left\||v|^{2} f_{n}\right\|+\left\|\left(-\frac{1}{4} \frac{d^{2}}{d y^{2}}-\mu^{2}\right) \phi_{n}\right\|+\frac{1}{2}\left\|\overline{\left(v^{\prime}-\alpha v\right)} f_{n}\right\| \\
& \quad \rightarrow 0 \text { as } n \rightarrow \infty .
\end{aligned}
$$

In the same way, the second term on the right hand side of (3.2) converges to 0 as $n \rightarrow \infty$. Hence

$$
\left\|\left(H_{-}-2 \mu^{2}\right) \Phi_{n}\right\| \rightarrow 0 \text { as } n \rightarrow \infty
$$

Therefore, for every $\mu \geq 0,2 \mu^{2} \in \sigma\left(H_{-}\right)$. Since $H_{-}$is non-negative self-adjoint, $\sigma\left(H_{-}\right)=$ $[0, \infty)$. It follows in the same way as in the proof of Theorem 3.1 that $\sigma\left(H_{+}\right)=[0, \infty)$.

Corollary 3.5. Suppose $V \in \mathcal{V}_{1}$. Then $H_{ \pm}$is not semi-Fredholm.

Proof. We can prove this corollary in the same way as in the proof of Corollary 3.2 .

Corollary 3.6. The operator $Q_{+}$is not semi-Fredholm.

Proof. We have $\sigma\left(Q_{+}^{*} Q_{+}\right)=\sigma\left(H_{+}\right)=[0, \infty)$. Hence $\inf \sigma\left(Q_{+}^{*} Q_{+}\right) \backslash\{0\}=0$. It implies that $Q_{+}$is not semi-Fredholm.

Remark 3.7. We have index $Q_{+}=\operatorname{dim} \operatorname{ker} H_{+}-\operatorname{dim} \operatorname{ker} H_{-}$. By Corollary 3.6, index $Q_{+}$may be unstable under perturbations. This suggests that ker $H_{-}$may be also unstable under perturbations. 


\section{Zero-Energy Ground States of the Bosonic Hamiltonian}

In this section we discuss zero-energy ground states of the bosonic Hamiltonian $H_{+}$and the structure of them, and give a concrete class of superpotentials with which the models have infinitely many bosonic zero-energy ground states.

\section{A. Symmetry Group and Direct Integral Decomposition.}

Let $\partial / \partial y$ be the generalized derivative and put

$$
N_{+}=i \frac{\partial}{\partial y}+\frac{\alpha}{2} \sigma_{3}
$$

with $D\left(N_{+}\right)=D(\partial / \partial y) \subset \mathcal{H}_{+}$, where $\sigma_{3}=\left(\begin{array}{cc}1 & 0 \\ 0 & -1\end{array}\right)$. Then $N_{+}$is self-adjoint. Hence, by Stone's theorem it generates the strongly continuous one parameter unitary group $\left\{e^{i s N_{+}} \mid s \in \mathbb{R}\right\}$ on $\mathcal{H}_{+}$.

Lemma 4.1. Let $V \in \mathcal{V}_{0}$. Then, for all $t, s \in \mathbb{R}$,

$$
e^{i t H_{+}} e^{i s N_{+}}=e^{i s N_{+}} e^{i t H_{+}}
$$

Proof. We can prove this lemma in the same way as in the proof of Lemma 3.1 in Ref. 6.

Remark. In the case $V(z)=\lambda z^{p}=\lambda r^{p} e^{i p \theta}$ (polar coordinate; $z=r e^{i \theta}$ ), the generator of a symmetry group of $H_{+}$is given by $L=i \partial / \partial \theta+(p-2) \sigma_{3} / 2$. In the case $V(z)=\lambda / z^{p}=$ $\lambda r^{p} e^{-i p \theta}$, the operator $M=i \partial / \partial \theta-(p+2) \sigma_{3} / 2$ is the generator of a symmetry group of $H_{+}$. Both of the spectrum of $L$ and of $M$ are equal to $\{n-p / 2 \mid n \in \mathbb{N}\}$. (See Refs.4 and 5.)

The generator $N_{+}$has no point spectrum and the spectrum of $N_{+}$is equal to $\mathbb{R}$. This is different from the case of $L$ and $M$. However every $p \in \mathbb{R}$ is a generalized eigenvalue of $N_{+}$ and the generalized eigenspace with eigenvalue $p$ is given by

$$
\mathcal{H}_{p}=\left\{\left(\begin{array}{c}
\phi(x) e^{-i(p-\alpha / 2) y} \\
\psi(x) e^{-i(p+\alpha / 2) y}
\end{array}\right) \mid \phi, \psi \in L^{2}(\mathbb{R})\right\} \cong \stackrel{2}{\oplus} L^{2}(\mathbb{R})
$$

This suggests that the bosonic Hilbert space $\mathcal{H}_{+}$may be written as the constant fiber direct integral over $\mathbb{R}$ with fiber $\oplus^{2} L^{2}(\mathbb{R})$ and $H_{+}$may be decomposable with respect to the direct integral decomposition. In fact, this is true as we show in the following. 
The operator $U: L^{2}\left(\mathbb{R}^{2} ; \mathbb{C}^{2}\right) \rightarrow L^{2}\left(\mathbb{R} ; \oplus^{2} L^{2}(\mathbb{R})\right)$ given by

$$
U\left(\begin{array}{l}
f \\
g
\end{array}\right)(p)=\left(\begin{array}{c}
(2 \pi)^{-1 / 2} \int_{\mathbb{R}} e^{-i p y} f(y, x) d y \\
(2 \pi)^{-1 / 2} \int_{\mathbb{R}} e^{-i(p-\alpha) y} g(y, x) d y
\end{array}\right), \quad\left(\begin{array}{l}
f \\
g
\end{array}\right) \in L^{2}\left(\mathbb{R}^{2} ; \mathbb{C}^{2}\right),
$$

is unitary. Then this operator gives a direct integral decomposition of $L^{2}\left(\mathbb{R} ; \oplus^{2} L^{2}(\mathbb{R})\right)$. We denote by $f^{\prime}$ the derivative of $f$ with respect to $x$.

Lemma 4.2. Let $V \in \mathcal{V}_{0}$ with $\partial V(z)=v(x) e^{i \alpha y}$. Let

$$
h(p)=\left(\begin{array}{cc}
-\frac{1}{4} \frac{d^{2}}{d x^{2}}+|v|^{2}+\frac{1}{4} p^{2} & -\frac{i}{2}\left(v^{\prime}+\alpha v\right) \\
\frac{i}{2} \overline{\left(v^{\prime}+\alpha v\right)} & -\frac{1}{4} \frac{d^{2}}{d x^{2}}+|v|^{2}+\frac{1}{4}(p-\alpha)^{2}
\end{array}\right)
$$

acting in $\oplus^{2} L^{2}(\mathbb{R})$. Then

$$
U H_{+} U^{*}=\int_{\mathbb{R}}^{\oplus} h(p) d p .
$$

Proof. Let $\mathcal{D}=C_{0}^{\infty}\left(\mathbb{R} ; \mathbb{C}^{2}\right)$ and $A_{p}$ be an operator given by

$$
A_{p}=\left(\begin{array}{cc}
-i \bar{v} & \frac{1}{2}\left(\frac{d}{d x}+p-\alpha\right) \\
\frac{1}{2}\left(\frac{d}{d x}-p\right) & i v
\end{array}\right)
$$

with $D\left(A_{p}\right)=\mathcal{D}$. The operator

$$
T=\left(\begin{array}{cc}
0 & A_{p} \\
A_{p}^{*} & 0
\end{array}\right)
$$

can be written

$$
T=\frac{1}{2}\left(\begin{array}{cccc}
0 & 0 & 0 & 1 \\
0 & 0 & 1 & 0 \\
0 & -1 & 0 & 0 \\
-1 & 0 & 0 & 0
\end{array}\right) \frac{d}{d x}+B
$$

with $D(T)=\mathcal{D} \oplus \mathcal{D}$ and $B$ being a $C^{\infty} 4 \times 4$ matrix-valued function. It is obvious that $T$ is symmetric on $D(T)$. By Theorem 2.2 in Ref.8,

$$
T^{2}=\left(\begin{array}{cc}
A_{p} A_{p}^{*} & 0 \\
0 & A_{p}^{*} A_{p}
\end{array}\right)
$$

is essentially self-adjoint on $\mathcal{D} \oplus \mathcal{D}$. Hence $A_{p}^{*} A_{p}$ is essentially self-adjoint on $\mathcal{D}$. Since $h(p)=$ $A_{p}^{*} A_{p}$ on $\mathcal{D}, h(p)$ is essentially self-adjoint on $\mathcal{D}$. The operator $R$ given by

$$
R=\int_{\mathbb{R}}^{\oplus}\{h(p)+i\}^{-1} d p
$$


is bounded and decomposable on $L^{2}\left(\mathbb{R} ; \oplus^{2} L^{2}(\mathbb{R})\right)$. Moreover we have $R=\left(U H_{+} U^{*}+i\right)^{-1}$. Hence $U H_{+} U^{*}$ is decomposable and has the representation (4.1).

The following lemma will be needed.

Lemma 4.3. Let $\langle M, \mu\rangle$ be a $\sigma$-finite measure space. Let $\mathcal{H}$ and $\mathcal{K}$ be separable Hilbert spaces and $C(\mathcal{H}, \mathcal{K})$ be the set of densely defined closed linear operators from $\mathcal{H}$ to $\mathcal{K}$. Let $A(m)$ be a $C(\mathcal{H}, \mathcal{K})$-valued, measurable function on $M$. Let $H(m)=A(m)^{*} A(m), m \in M$, and

$$
H=\int_{M}^{\oplus} H(m) d \mu
$$

which acts in $\int_{M}^{\oplus} \mathcal{H} d \mu$, the constant fiber direct integral of $\mathcal{H}$. Then

$$
\operatorname{ker} H=\int_{M}^{\oplus} \operatorname{ker} A(m) d \mu .
$$

Proof. In general, $\operatorname{ker} A^{*} A=\operatorname{ker} A$. By the general theory of constant fiber direct integral, $\operatorname{ker} H=\int_{M}^{\oplus} \operatorname{ker} H(m) d \mu$. Hence we obtain the desired result.

The identity $\operatorname{ker} A^{*} A=\operatorname{ker} A$ implies that $\operatorname{ker}\left(U H_{+} U^{*}\right)=\operatorname{ker}\left(U Q_{+} U^{*}\right)$.

Lemma 4.4. Let $V \in \mathcal{V}_{0}$ with $\partial V(z)=v(x) e^{i \alpha y}$. Let $\Phi \in \operatorname{ker}\left(U Q_{+} U^{*}\right)$. Write $\Phi(p)=(\phi(p, \cdot),-i \psi(p, \cdot))$. Then the following equations hold a.e. (almost everywhere) $p$ in the generalized sense:

$$
\begin{aligned}
& \overline{v(\cdot)} \phi(p, \cdot)+\frac{1}{2}\left(\frac{\partial}{\partial x}+p-\alpha\right) \psi(p, \cdot)=0, \\
& \frac{1}{2}\left(\frac{\partial}{\partial x}-p\right) \phi(p, \cdot)+v(\cdot) \psi(p, \cdot)=0 .
\end{aligned}
$$

Proof. By direct computations.

We fix $p$ to solve the equation $U Q_{+} U^{*} \Phi=0$ on each of fiber spaces. We rewrite $(\phi(p, \cdot), \psi(p, \cdot))$ as $((\phi(\cdot), \psi(\cdot))$.

Lemma 4.5. Let $V \in \mathcal{V}_{0}$ with $\partial V(z)=v(x) e^{i \alpha y}$. Suppose $v^{\prime} / v \in C^{\infty}(\mathbb{R})$. 
(i) Every solution $(\phi, \psi)$ of (4.2) and (4.3) is a $C^{\infty}$-function and satisfies the following ordinary differential equations:

$$
\begin{aligned}
& -\phi^{\prime \prime}+\left(\frac{v^{\prime}}{v}+\alpha\right) \phi^{\prime}+\left(4|v|^{2}-p \frac{v^{\prime}}{v}+p(p-\alpha)\right) \phi=0 \\
& -\psi^{\prime \prime}+\left(\frac{\bar{v}^{\prime}}{\bar{v}}+\alpha\right) \psi^{\prime}+\left(4|v|^{2}+(p-\alpha) \frac{\bar{v}^{\prime}}{\bar{v}}+p(p-\alpha)\right) \psi=0 \\
& \frac{1}{2}\left(\frac{d}{d x}-p\right) \phi+v \psi=0
\end{aligned}
$$

(ii) For every solution $\phi$ of (4.4), the function $\psi$ satisfying (4.6) is a solution of (4.5).

Proof. Since $v$ and $v^{\prime} / v$ are in $C^{\infty}(\mathbb{R})$, it follows from elliptic regularity that $\phi$ and $\psi$ are in $C^{\infty}(\mathbb{R})$. Therefore we have the desired results.

\section{B. Zero-energy states in each of fiber spaces.}

We introduce a subclass of superpotentials in $\mathcal{V}_{0}$.

Definition 4.6. We say that $V$ is in $\mathcal{V}_{2}$ if there exist a constant $\lambda \in \mathbb{C} \backslash\{0\}$ and $v \in C^{\infty}(\mathbb{R})$ such that $v>0, \partial V(z)=\lambda v(x) e^{i \alpha y}$ and

$$
\varliminf_{x \rightarrow \infty} e^{\alpha x} v(x)>0
$$

Let $V \in \mathcal{V}_{2}$. It is well known that the equations (4.4) and (4.5) can be transformed into the equations of the form

$$
y^{\prime \prime}-G y=0
$$

Let $h(x)=\sqrt{e^{\alpha x} v(x)}$ and define $f(x)=\phi(x) / h(x)$ and $g(x)=\psi(x) / h(x)$. Then we have from (4.4) and (4.5)

$$
\begin{aligned}
& f^{\prime \prime}-G_{p} f=0 \\
& g^{\prime \prime}-G_{\alpha-p} g=0,
\end{aligned}
$$

where

$$
G_{p}(x)=4|\lambda|^{2} v^{2}+\frac{1}{4}\left(\frac{v^{\prime}}{v}-2 p+\alpha\right)^{2}+\frac{1}{2}\left(\frac{v^{\prime}}{v}\right)^{2}-\frac{1}{2} \frac{v^{\prime \prime}}{v}
$$

We next investigate properties of solutions of the equation (4.8). 
Lemma 4.7. Suppose that $G \in C(\mathbb{R})$ and there exist constants $c_{-}, c_{+} \in \mathbb{R}$, positive constants $\delta, \gamma, \sigma$ and $\rho>1 / 2$ such that

(i) $\gamma^{2} \leq G(x) \leq \delta^{2} \quad$ on $\left(-\infty, c_{-}\right] \quad$ and $\quad \lim _{x \rightarrow-\infty} h(x) e^{(2 \delta-\gamma)|x|}|x|^{\rho}=0$,

(ii) $\sigma^{2} \leq G(x)$ on $\left[c_{+}, \infty\right)$ and $\lim _{x \rightarrow \infty} h(x) e^{-\sigma x} x^{\rho}=0$.

Then there exists a unique solution $y$ of (4.8), up to constant multiples, such that hy $\in L^{2}(\mathbb{R})$.

Proof. By Lemma A.2 (see Appendix), there exists one and only one solution $y_{+}$of (4.8) on $\left(-\infty, c_{-}\right]$such that

$$
y_{+}\left(c_{-}\right)=1 \text { and } y_{+}(x) \rightarrow 0 \quad \text { as } \quad x \rightarrow-\infty
$$

Moreover, by Lemma A.4 the following estimate holds:

$$
e^{-\delta\left|x-c_{-}\right|} \leq y_{+}(x) \leq e^{-\gamma\left|x-c_{-}\right|} \text {for } x \leq c_{-}
$$

Let

$$
z(x)=y_{+}(x) \int_{c_{-}}^{x} y_{+}(t)^{-2} d t \quad \text { for } \quad x \leq c_{-} .
$$

Then $z$ is a solution of (4.8) and linearly independent of $y_{+} \cdot(4.11)$ gives the following estimate:

$$
|z(x)| \leq y_{+}(x)\left|\int_{c_{-}}^{x} y_{+}(t)^{-2} d t\right| \leq \text { const. } e^{(2 \delta-\gamma)|x|}
$$

Since all the solutions $y$ of (4.8) are linear combinations of $y_{+}$and $z$, it follows that $h y \in L^{2}\left(-\infty, c_{-}\right)$.

By. Lemma A.4 there exists a unique subdominant solution $y_{+}$of (4.8), up to constant multiples, on $\left[c_{+}, \infty\right)$ such that

$$
0<y_{+}(x)<\text { const. } e^{-\sigma x} \text { for } x \geq c_{+}
$$

Obviously we have $h y_{+} \in L^{2}\left(c_{+}, \infty\right)$. On the other hand, by Lemma A.3 any solution $z$ of (4.8) linearly independent of $y_{+}$tends to infinity as $x \rightarrow \infty$. Since $\underset{x \rightarrow \infty}{\lim } h(x)>0$ by (4.7), it follows that $h z \notin L^{2}\left(c_{+}, \infty\right)$. Consequently, there exists a unique solution $y$ of (4.8) on $\left[c_{+}, \infty\right)$ such that $h y \in L^{2}\left(c_{+}, \infty\right)$. Since every solution of $(4.8)$ is continuous on $\left[c_{-}, c_{+}\right]$, it follows that there exists a unique solution $y$ of (4.8) such that $h y \in L^{2}(\mathbb{R})$.

We are now ready to solve the equations (4.4)-(4.6). 
Lemma 4.8. Let $V \in \mathcal{V}_{2}$. Suppose that the functions $G_{p}$ and $G_{\alpha-p}$ satisfy the hypothesis in Lemma 4.7 with $G=G_{p}, G=G_{\alpha-p}$. Suppose that $\varlimsup_{x \rightarrow \infty} e^{\alpha x} v^{-1}(x)<\infty$ and $\varlimsup_{x \rightarrow \infty}\left|v^{\prime}(x) v^{-2}(x)\right|<\infty$. Then there exists a unique solution of $(4.4)-(4.6)$ in $\oplus^{2} L^{2}(\mathbb{R})$, up to constant multiples.

Proof. By Lemma 4.7 we can construct a unique solution $\phi=h f$ of $(4.4)$ in $L^{2}(\mathbb{R})$. We define $\psi$ by (4.6) with this $\phi$. Then by part (ii) of Lemma $4.5, \psi$ is a solution of (4.5). We can prove in the same way as in the proof of Lemma 4.7 that $\psi$ is in $L^{2}\left(-\infty, c_{-}\right)$. Since $\phi=h f$ and $h=\sqrt{e^{\alpha x} v}$

$$
-2 \lambda \psi=\frac{1}{v}\left(\frac{d}{d x}-p\right) \phi=\frac{1}{2}\left[\frac{\alpha-2 p}{v}+\frac{v^{\prime}}{v^{2}}\right] h f+\sqrt{\frac{e^{\alpha x}}{v}} f^{\prime}
$$

holds. By the construction of $\phi$, the function $f$ is a subdominant solution of (4.9). Hence, by Lemma A.2, $f^{\prime} \in L^{2}\left(c_{+}, \infty\right)$ and by Lemma $A .4, f \in L^{2}\left(c_{+}, \infty\right)$. Hence it follows that all the terms on the right hand side of $(4.12)$ are in $L^{2}\left(c_{+}, \infty\right)$. Consequently, $\psi \in L^{2}(\mathbb{R})$. By Lemma 4.7 , this $\psi$ is a unique solution of $(4.5)$ in $L^{2}(\mathbb{R})$. Hence the pair $(\phi, \psi)$ is a unique solution of (4.4)-(4.6) in $\oplus^{2} L^{2}(\mathbb{R})$.

\section{Zero-energy states in the whole space.}

In the last subsection we got a zero-eigenvalue solution of $U H_{+} U^{*} \Phi=0$ restricted to each fiber space. By bundling them, we shall construct solutions of $U H_{+} U^{*} \Phi=0$. But it is not obvious whether the bundled function is Lebesgue measurable on $\mathbb{R}$ or not. In this subsection we first examine this measurability and then prove that $\operatorname{dim} \operatorname{ker} H_{+}=\infty$. The following is the main theorem in this section.

Theorem 4.9. Let $V \in \mathcal{V}_{2}$. Suppose that there exists an interval $\mathrm{I}$ of $\mathbb{R}$ with non-zero Lebesgue measure, satisfying the following properties:

(i) there exist $c_{-}, c_{+}, \delta, \gamma, \sigma$ and $\rho$ such that for all $p \in \mathrm{I}, G=G_{p}$ satisfies the hypothesis in Lemma 4.7;

(ii) there exist $c_{-}, c_{+}, \delta, \gamma, \sigma$ and $\rho$ such that for all $p \in \mathbf{I}, G=G_{\alpha-p}$ satisfies the hypothesis in Lemma 4.7;

(iii) $\varlimsup_{x \rightarrow \infty} e^{\alpha x} v^{-1}(x)<\infty$ and $\varlimsup_{x \rightarrow \infty}\left|v^{\prime}(x) v^{-2}(x)\right|<\infty$.

Then

$$
\operatorname{dim} \operatorname{ker} H_{+}=\infty
$$


The proof of this theorem is based on the following proposition.

Proposition 4.10. Suppose that hypothesis (i) for $G_{p}$ in Theorem 4.9 is satisfied. Then there exists a solution $y_{p}$ of

$$
y^{\prime \prime}-G_{p} y=0 \text { on } \mathbf{I} \times \mathbb{R}
$$

such that $h(\cdot) y_{p}(\cdot) \in L^{2}(\mathbb{R})$ for every $p \in \mathbf{I}$ and $h y_{p}: \mathbf{I} \rightarrow L^{2}(\mathbb{R}), p \mapsto h(\cdot) y_{p}(\cdot)$ is measurable. Moreover if the measure of $\mathbf{I}$ is finite, then $h y_{p} \in L^{2}\left(\mathbf{I} ; L^{2}(\mathbb{R})\right)$.

Remark. The function $h y_{p}: \mathrm{I} \rightarrow L^{2}(\mathbb{R})$ is measurable if and only if for all $g \in L^{2}(\mathbb{R})$ the mapping I $\ni p \mapsto\left\langle h y_{p}, g\right\rangle_{L^{2}(\mathbb{R})} \in \mathbb{C}$ is measurable. (See Ref. 9, vol. I.)

Proof. Without loss of generality we can assume I is a bounded closed interval.

Step 1. We prove the continuity of the functions $y_{p}$ and $y_{p}^{\prime}$ on $\mathrm{I} \times \mathbb{R}$.

We assume $y_{p}\left(c_{+}\right)=1$ for every $p \in \mathbf{I}$. For each $p \in \mathbf{I}$, let $y_{p, 1}$ and $y_{p, 0}$ be the solutions of (4.13) with $y_{p, 1}\left(c_{+}\right)=1, y_{p, 1}^{\prime}\left(c_{+}\right)=0, y_{p, 0}\left(c_{+}\right)=0$ and $y_{p, 0}^{\prime}\left(c_{+}\right)=1$. We put

$$
\alpha(p)=-\lim _{x \rightarrow \infty} \frac{y_{p, 1}(x)}{y_{p, 0}(x)}
$$

From the proof of Lemma 4.7, $y_{p}$ must be the normalized subdominant solution of (4.13) on $\left(c_{+}, \infty\right)$. By Lemma A.2, we can write

$$
y_{p}(x)=y_{p, \mathbf{1}}(x)+\alpha(p) y_{p, 0}(x)
$$

The function $G_{p}(x)$ is continuous in $x \in \mathbb{R}$ and we have for all $p, q \in \mathbb{R}$

$$
G_{p}(x)-G_{q}(x)=(q-p)\left(\frac{v^{\prime}(x)}{v(x)}+\alpha-p-q\right)
$$

Since $v^{\prime} / v$ is continuous and $\mathbf{I}$ is bounded, $G_{p}$ satisfies the Lipschitz condition with respect to $p$ on every compact interval of $\mathbb{R}$. Hence, by a general theory of ordinary differential equation, the solutions $y_{p, 1}, y_{p, 1}^{\prime}, y_{p, 0}$ and $y_{p, 0}^{\prime}$ are continuous in $p$ at each $x \in \mathbb{R}$. (See e.g. Ref. 10.)

Claim. Let $c \in(0, \infty)$. We consider a family of $C^{1}$-functions such that

$$
\left\{f_{\alpha}:(0, c) \rightarrow \mathbb{R} \mid f_{\alpha}^{\prime} \geq 0, \exists \lim _{x \rightarrow c} f_{\alpha}(x)\right\}_{\alpha \in A}
$$


If there exists a function $F:(0, c) \rightarrow \mathbb{R}$ such that $F(x) \geq f_{\alpha}^{\prime}(x)$ on $(0, c)$ for every $\alpha \in A$ and $\int_{0}^{c} F(t) d t<\infty$, then the convergence, $\lim _{x \rightarrow c} f_{\alpha}(x)=f_{\alpha}(c)$, is uniform in $\alpha \in A$.

Proof. For every $\alpha \in A$, we have

$$
0 \leq f_{\alpha}(c)-f_{\alpha}(t)=\int_{t}^{c} f_{\alpha}^{\prime}(s) d s \leq \int_{t}^{c} F(s) d s .
$$

As $t \rightarrow c$, the right hand side of this inequality converges to 0 uniformly in $\alpha$. Hence the desired result follows.

Since $y_{p, 0}>0$ by Lemma A.1, the function $y_{p, 1} / y_{p, 0}$ is differentiable on $\left(c_{+}, \infty\right)$. We have

$$
\frac{d}{d x}\left(\frac{y_{p, 1}}{y_{p, 0}}\right)=\frac{W\left(y_{p, 1}, y_{p, 0}\right)}{\left(y_{p, 0}\right)^{2}}=\left(\frac{1}{y_{p, 0}}\right)^{2}>0
$$

where $W$ is Wronskian. For the solution $Y$ of $y^{\prime \prime}-\sigma^{2} y=0$ with $Y\left(c_{+}\right)=0$ and $Y^{\prime}\left(c_{+}\right)=1$, Lemma A.4 implies that $0<Y(x) \leq y_{p, 0}$ on $\left(c_{+}, \infty\right)$ for every $p \in \mathbf{I}$. Hence

$$
\left(\frac{1}{Y(x)}\right)^{2} \geq \frac{d}{d x}\left(\frac{y_{p, 1}}{y_{p, 0}}\right)
$$

Since $Y(x)=\sinh \left(\sigma\left(x-c_{+}\right)\right) / \sigma$, we have

$$
\int_{c_{+}+1}^{\infty}\left(\frac{1}{Y(x)}\right)^{2} d x<\infty
$$

By the above claim, $-y_{p, 1}(x) / y_{p, 0}(x)$ converges to $\alpha(p)$ as $x \rightarrow \infty$ uniformly in $p \in \mathbf{I}$. Since $y_{p, 1}(x) / y_{p, 0}(x)$ is continuous in $p$ at each $x \in \mathbb{R}, \alpha(p)$ is continuous. Consequently, $y_{p}$ and $y_{p}^{\prime}$ are continuous in $p$ and $x$. This fact will be used also in the proof of Theorem 4.9.

Step 2. We prove the measurability of $h y_{p}$. To do this, we construct a function $F$ on $\mathbb{R}$ such that $h F \in L^{2}(\mathbb{R})$ and $\left|y_{p}\right| \leq|F|$ on $\mathbb{R}$ for every $p \in \mathbb{I}$. Then, it follows from Step 1 and Lebesgue dominate convergence theorem that for all $g \in L^{2}(\mathbb{R}),\left\langle h y_{p}, g\right\rangle$ is continuous in $p$.

On the interval $\left(c_{+}, \infty\right), y_{p}$ is the normalized subdominant solution of (4.13) for each $p$. Hence we define $F$ on $\left(c_{+}, \infty\right)$ to be the normalized subdominant solution of $y^{\prime \prime}-\sigma^{2} y=0$. Then, by Lemma A.4, we have

$$
0 \leq y_{p}(x) \leq F(x) \leq e^{-\sigma\left(x-c_{+}\right)} \text {on }\left[c_{+}, \infty\right)
$$

It is clear that $h F \in L^{2}\left(c_{+}, \infty\right)$. 
Let $M=\sup \left\{\left|y_{p}(x)\right| ; p \in \mathrm{I}, c_{-} \leq x \leq c_{+}\right\}$and $N=\sup \left\{\left|y_{p}\left(c_{-}\right)\right| ; p \in \mathrm{I}\right\}$. Since $y_{p}$ and $y_{p}^{\prime}$ are continuous in $p$ and $x$ and $\mathbf{I}$ is bounded, $M$ and $N$ are finite.

We put $F(x)=M$ on $\left(c_{-}, c_{+}\right)$. Then $h F \in L^{2}\left(c_{-}, c_{+}\right)$and $\left|y_{p}\right| \leq F$.

For each $p \in \mathrm{I}$, there exists the normalized subdominant solution $z_{p}$ of $(4.13)$ on $\left(-\infty, c_{-}\right)$. The solution $w_{p}$ of (4.13) given by

$$
w_{p}(x)=z_{p}(x) \int_{c_{-}}^{x} z_{p}(t)^{-2} d t
$$

is linearly independent of $z_{p}$. Then $w_{p}\left(c_{-}\right)=0$ and $w_{p}^{\prime}\left(c_{-}\right)=z_{p}\left(c_{-}\right)^{-1}=1$. Hence we have

$$
y_{p}(x)=y_{p}\left(c_{-}\right) z_{p}(x)+\left\{y_{p}^{\prime}\left(c_{-}\right)-y_{p}\left(c_{-}\right) z_{p}^{\prime}\left(c_{-}\right)\right\} w_{p}(x)
$$

Lemma A.4 implies

$$
e^{\delta\left(x-c_{-}\right)} \leq z_{p}(x) \leq e^{\gamma\left(x-c_{-}\right)} .
$$

We obtain

$$
\begin{aligned}
\left|w_{p}(x)\right| & \leq e^{\gamma\left(x-c_{-}\right)}\left|\int_{c_{-}}^{x} e^{-2 \delta\left(t-c_{-}\right)} d t\right| \\
& =\frac{1}{2 \delta} e^{(2 \delta-\gamma) c_{-}} e^{\gamma x}\left(e^{-2 \delta x}-e^{-2 \delta c_{-}}\right) .
\end{aligned}
$$

These estimates do not depend on $p$. In the same way as in Step 1, we see that $z_{p}^{\prime}\left(c_{-}\right)$is continuous in $p$. Hence, the quantity $L=\sup \left\{\left|z_{p}^{\prime}\left(c_{-}\right)\right| ; p \in \mathrm{I}\right\}$ is finite. Consequently, we have for every $p \in \mathbf{I}$

$$
\left|y_{p}(x)\right| \leq M e^{\gamma\left(x-c_{-}\right)}+(N+M L) \frac{1}{2 \delta} e^{(2 \delta-\gamma) c_{-}} e^{\gamma x}\left(e^{-2 \delta x}-e^{-2 \delta c_{-}}\right) .
$$

Hence there exists a constant $C$ not depending on $p$ such that

$$
\left|y_{p}(x)\right| \leq C e^{(\gamma-2 \delta) x} \quad \text { on } \quad\left(-\infty, c_{-}\right]
$$

We put $F(x)=C e^{(\gamma-2 \delta) x}$ on $\left(-\infty, c_{-}\right]$. By the hypothesis of this proposition, $h F \in$ $L^{2}\left(-\infty, c_{-}\right)$.

In this way we obtain a function $F$ on $\mathbb{R}$ such that $h F \in L^{2}(\mathbb{R})$ and $\left|y_{p}\right| \leq|F|$. Hence, the function $\mathbf{I} \ni p \mapsto h f_{p} \in L^{2}(\mathbb{R})$ is measurable. Since $\mathbf{I}$ is bounded, $h F \in L^{2}\left(\mathbf{I} ; L^{2}(\mathbb{R})\right)$. Hence $h y_{p} \in L^{2}\left(\mathbf{I} ; L^{2}(\mathbb{R})\right)$. 
Remark 4.11. It is easy to see that it is sufficient to assume, in Step 1 , that $y_{p}\left(c_{+}\right)$is continuous in $p$. Then we have $y_{p}(x)=y_{p}\left(c_{+}\right)\left\{y_{p, 1}(x)+\alpha(p) y_{p, 0}(x)\right\}$.

Proof of Theorem 4.9. By Lemma 4.8, for each $p \in \mathbf{I}$, there exists a unique solution $(\phi(p, \cdot), \psi(p, \cdot))$ of $(4,4)-(4.6)$, up to constant multiples. We normalize the solution with $\phi\left(p, c_{+}\right)=1$. By Step 1 in the proof of Proposition 4.10, $\phi(p, x)$ and $\phi^{\prime}(p, x)$ are continuous in $\mathrm{I} \times \mathbb{R}$. Hence by $(4.6), \psi\left(p, c_{+}\right)$is continuous in $p \in \mathrm{I}$, too. By Proposition 4.10 together with Remark 4.11, the function I $\ni p \mapsto(\phi, \psi) \in \oplus^{2} L^{2}(\mathbb{R})$ is measurable.

We can decompose the interval I into infinitely many subintervals $\left\{\mathbf{I}_{n}\right\}_{n \in \mathbb{N}}$ such that the measure of $\mathbf{I}_{n}$ is non-zero. For each $n \in \mathbb{N}$ we define a $\oplus^{2} L^{2}(\mathbb{R})$-valued function $\Phi_{n}$ on $\mathbb{R}$ by

$$
\Phi_{n}(p)= \begin{cases}\left(\begin{array}{c}
\phi(p, \cdot) \\
-i \psi(p, \cdot)
\end{array}\right), & \text { if } p \in \mathbf{I}_{n} \\
0, & \text { otherwise. }\end{cases}
$$

Then, by Proposition $4.10, \Phi_{n}$ is in $L^{2}\left(\mathbb{R} ; \oplus^{2} L^{2}(\mathbb{R})\right)$ and by the construction of $\Phi_{n}$, we have $U H_{+} U^{*} \Phi_{n}=0$. It is clear that for $n \neq m \Phi_{n}$ is orthogonal to $\Phi_{m}$. Consequently, it follows that $\operatorname{dim} \operatorname{ker} U H_{+} U^{*}=\infty$.

Remark 4.12. From the discussions in this section, we can see that there exist infinitely many generalized zero-energy states of $H_{+}$. For the generalized eigenvector

$$
\left(\begin{array}{c}
f(x) e^{-i(p-\alpha / 2) y} \\
-i g(x) e^{-i(p+\alpha / 2) y}
\end{array}\right)
$$

of $N_{+}$with eigenvalue $p$, we have

$$
Q_{+} \Psi=\left(\begin{array}{c}
-i e^{-i(p+\alpha / 2) y}\left(\bar{\lambda} v(x) f(x)+\frac{1}{2}\left(\frac{\partial}{\partial x}-p-\frac{\alpha}{2}\right) g(x)\right) \\
e^{-i(p-\alpha / 2) y}\left(\frac{1}{2}\left(\frac{\partial}{\partial x}+p-\frac{\alpha}{2}\right) f(x)+\lambda v(x) g(x)\right)
\end{array}\right)
$$

Hence for every solution $(f, g)$ of

$$
\bar{\lambda} v f+\frac{1}{2}\left(\frac{\partial}{\partial x}-p-\frac{\alpha}{2}\right) g=0 \quad \text { and } \quad \frac{1}{2}\left(\frac{\partial}{\partial x}+p-\frac{\alpha}{2}\right) f+\lambda v g=0
$$

the vector $\Psi=\left(f(x) e^{-i(p-\alpha / 2) y},-i g(x) e^{-i(p+\alpha / 2) y}\right)$ satisfies the equation $H_{+} \Psi=Q_{+}^{*} Q_{+} \Psi=$ 0 . But $\Psi \notin L^{2}\left(\mathbb{R}^{2}\right)$. It means that $\Psi$ is a generalized zero-energy eigenvector of $H_{+}$. Since every $p \in \mathbb{R}$ is a generalized eigenvalue of $N_{+}, H_{+}$has infinitely many generalized zero-energy states. This kind of phenomenon occurs also in the $\mathrm{N}=2 \mathrm{WZ}$ model with $V(z)=\lambda z^{p}$ and $V(z)=\lambda / z^{p} \cdot{ }^{4,5}$ 


\section{A Concrete Class of Superpotentials.}

In this subsection we give a concrete class of superpotentials, which satisfy the hypotheses of Theorems 4.9. In other words, we give a class of the $\mathrm{N}=2 \mathrm{WZ}$ type models which have infinitely many bosonic zero-energy ground states.

Definition 4.13. We say that $V: \mathbb{C} \rightarrow \mathbb{C}$ is in $\mathcal{V}_{3}$ if there exist constants $\lambda \in \mathbb{C} \backslash\{0\}, \beta \in \mathbb{R}$ with $\beta \geq \alpha$ and $w \in C^{\infty}(\mathbb{R})$ such that:

(1) $\quad \partial V(z)=\lambda(1+w(x)) e^{\beta x} e^{i \alpha y}$

(2) $1+w>0$ on $\mathbb{R}$,

(3) $\quad w(x) \rightarrow 0$ as $x \rightarrow \infty$,

(4) $w(x), w^{\prime}(x)$ and $w^{\prime \prime}(x) \rightarrow 0$ as $x \rightarrow-\infty$,

(5) $\quad w^{\prime} /(1+w)$ and $w^{\prime \prime} /(1+w)$ are bounded on $\mathbb{R}$.

It is clear that $\mathcal{V}_{3} \subset \mathcal{V}_{1} \cap \mathcal{V}_{2}$

Theorem 4.14. Let $V \in \mathcal{V}_{3}$. Then

$$
\operatorname{dim} \operatorname{ker} H_{+}=\infty
$$

Proof. We must prove that every function in $\mathcal{V}_{3}$ satisfies the hypotheses of Theorems 4.9. Since $V \in \mathcal{V}_{3}$, we can write $\partial V(z)=\lambda(1+w(x)) e^{\beta x} e^{i \alpha y}$. Let $I=(0, \alpha)$. We have

$$
\begin{aligned}
G_{p}(x)=4|\lambda|^{2}(1+w)^{2} e^{2 \beta x}+\frac{1}{4}\left(\alpha+\beta-2 p+\frac{w^{\prime}}{1+w}\right)^{2} \\
+\frac{1}{2}\left(\frac{w^{\prime}}{1+w}+\beta\right)^{2}-\frac{1}{2}\left(\frac{w^{\prime \prime}}{1+w}+2 \beta \frac{w^{\prime}}{1+w}+\beta^{2}\right) .
\end{aligned}
$$

By (4.13.5), $G_{p}(x)$ tends to infinity as $x \rightarrow \infty$ uniformly in $p \in \mathrm{I}$. Hence there exist constants $c_{+}$and $\sigma>(\alpha+\beta) / 2$ such that for every $p \in \mathrm{I}, G_{p} \geq \sigma^{2}$ on $\left[c_{+}, \infty\right)$ and for all $\rho \geq 0$, by (4.13.3),

$$
h(x) e^{-\sigma x} x^{\rho}=\sqrt{1+w(x)} e^{(\alpha+\beta) x / 2} e^{-\sigma x} x^{\rho} \rightarrow 0 \quad \text { as } \quad x \rightarrow \infty
$$

By (4.13.4), $G_{p}(x) \rightarrow(\alpha+\beta-2 p)^{2} / 4$ as $x \rightarrow-\infty$ uniformly in $p \in \mathrm{I}$. Thus, for each $\theta \in(0,1)$ there exists a constant $c_{-}$such that for every $p \in \mathbf{I}$,

$$
\theta^{2}\left(\frac{\alpha+\beta-2 p}{2}\right)^{2} \leq G_{p}(x) \leq \frac{1}{\theta^{2}}\left(\frac{\alpha+\beta-2 p}{2}\right)^{2}, \quad x \in\left(-\infty, c_{-}\right] .
$$


We put $\gamma=\theta(\alpha+\beta-2 p) / 2$ and $\delta=\theta^{-1}(\alpha+\beta-2 p) / 2$. We have

$$
\frac{1}{\theta}-\frac{\theta}{2}>\frac{1}{2} \text { and } \quad \frac{1}{\theta}-\frac{\theta}{2} \rightarrow \frac{1}{2} \quad \text { as } \quad \theta \rightarrow 1 \text {. }
$$

Hence for every $\varepsilon>0$, there exists $\theta$ in $(0,1)$ such that

$$
\frac{1}{\theta}-\frac{\theta}{2}=\frac{1+\varepsilon}{2}
$$

Thus,

$$
2 \delta-\gamma-\frac{\alpha+\beta}{2}=\frac{\varepsilon}{2}(\alpha+\beta)-(1+\varepsilon) p
$$

Since $p>0$, it follows that for all sufficiently small $\varepsilon, 2 \delta-\gamma-(\alpha+\beta) / 2<0$, which implies that for all $\rho \geq 0$

$$
h(x) e^{(2 \delta-\gamma)|x|}|x|^{\rho}=\sqrt{1+w(x)} e^{(\alpha+\beta) x / 2} e^{(2 \delta-\gamma)|x|}|x|^{\rho} \rightarrow 0 \quad \text { as } \quad x \rightarrow-\infty
$$

Hence hypothesis (i) of Theorem 4.9 is satisfied. Similarly we can show that hypothesis (ii) of Theorem 4.9 is satisfied. Hypothesis (iii) of Theorem 4.9 can easily be verified. We have thus proved Theorem 4.14.

It is obvious that $V(z)=\lambda e^{\alpha z}$ with $\lambda \in \mathbb{C} \backslash\{0\}$ is in $\mathcal{V}_{3}$. Hence we obtain the following result, which has been proved in Ref. 6 .

Corollary 4.15. Let $V(z)=\lambda e^{\alpha z}$ with $\lambda \in \mathbb{C} \backslash\{0\}$. Then

$$
\operatorname{dim} \operatorname{ker} H_{+}=\infty
$$

Example 4.16. Let $\mathcal{S}(\mathbb{R})$ be the set of rapidly decreasing $C^{\infty}$-functions on $\mathbb{R}$. Let

$$
S P=\left\{\begin{array}{l|l}
V: \mathbb{C} \rightarrow \mathbb{C} & \begin{array}{c}
\exists \lambda \in \mathbb{C} \backslash\{0\}, \exists \beta \geq \alpha, \exists s \in \mathcal{S}(\mathbb{R}) \\
\text { s.t.inf } \inf _{x \in \mathbb{R}}\{1+s(x)\}>0, \\
\partial V(z)=\lambda(1+s(x)) e^{\beta x} e^{i \alpha y}
\end{array}
\end{array}\right\}
$$

Then it is clear that every function in $S P$ is in $\mathcal{V}_{3}$. 


\section{The Vanishing Theorem}

In the last section, we formulated a condition under which dim ker $H_{+}=\infty$. In this section we consider a condition under which $\operatorname{dim} \operatorname{ker} H_{-}=0$. By Remark 3.7, we can not expect that the kernel of $H_{-}$is stable. Hence a class of $V$ for which ker $H_{-}=\emptyset$ may be very small.

We put

$$
F_{p}(x)=\frac{v^{\prime \prime}}{v}-\left(\frac{v^{\prime}}{v}\right)^{2}+2(p-\alpha)\left(\frac{v^{\prime}}{v}-p\right)
$$

Definition 5.1. We say that $V$ is in $\mathcal{V}_{4}$ if there exist a constant $\lambda \in \mathbb{C} \backslash\{0\}$ and $v \in C^{\infty}(\mathbb{R})$ such that $\partial V(z)=\lambda v(x) e^{i \alpha y}, v^{\prime} / v$ and $v^{\prime \prime} / v$ are bounded on $\mathbb{R}$ and $v^{\prime} / v \in C^{\infty}(\mathbb{R})$.

Theorem 5.2. Let $V \in \mathcal{V}_{4}$. Suppose that for a.e. $p \in \mathbb{R}$, either $F_{p} \leq 0$ on $\mathbb{R}$ or $F_{\alpha-p} \leq 0$ on $\mathbb{R}$ holds. Then,

$$
\operatorname{dim} \operatorname{ker} H_{-}=0 .
$$

To prove Theorem 5.2, we prepare some lemmas. We first note that there exists a symmetry group of the fermionic Hamiltonian $H_{-}$. Let

$$
N_{-}=i \frac{\partial}{\partial y}-\frac{\alpha}{2} \sigma_{3} \quad \text { with } \quad D\left(N_{-}\right)=D\left(\frac{\partial}{\partial y}\right) \subset \mathcal{H}_{-}
$$

Then the following lemma holds (see Lemma 4.1).

Lemma 5.3. Let $V \in \mathcal{V}_{0}$. Then for all $s, t \in \mathbb{R}$,

$$
e^{i s H_{-}} e^{i t N_{-}}=e^{i t N_{-}} e^{i s H_{-}} .
$$

The operator $\tilde{U}: L^{2}\left(\mathbb{R}^{2} ; \mathbb{C}^{2}\right) \rightarrow L^{2}\left(\mathbb{R} ; \oplus^{2} L^{2}(\mathbb{R})\right)$ given by

$$
\tilde{U}\left(\begin{array}{l}
f \\
g
\end{array}\right)(p)=\left(\begin{array}{c}
(2 \pi)^{-1 / 2} \int_{\mathbb{R}} e^{-i(p-\alpha) y} f(y, x) d y \\
(2 \pi)^{-1 / 2} \int_{\mathbb{R}} e^{-i p y} g(y, x) d y
\end{array}\right)
$$

is unitary. To identify the kernel of $H_{-}=Q_{+} Q_{+}^{*}$, we solve $\tilde{U} Q_{+}^{*} \tilde{U}^{*} \Phi=0$ on each of the fiber spaces of $L^{2}\left(\mathbb{R} ; \oplus^{2} L^{2}(\mathbb{R})\right)$ in a way similar to that in Sec. IV. 
Let $V \in \mathcal{V}_{4}$. Let $\Phi \in \operatorname{ker}\left(\tilde{U} H_{-} \tilde{U}^{*}\right)$ with $\Phi(p)=(\phi(p, \cdot),-i \psi(p, \cdot))$. In the same way as in the proof of Lemma 4.5 , we can see that for a.e. $p$ the following equations hold in the ordinary sense:

$$
\begin{aligned}
& -\phi^{\prime \prime}+\left(\frac{v^{\prime}}{v}-\alpha\right) \phi^{\prime}+\left(4|\lambda|^{2} v^{2}-(p-\alpha) \frac{v^{\prime}}{v}+p(p-\alpha)\right) \phi=0 \\
& -\psi^{\prime \prime}+\left(\frac{v^{\prime}}{v}-\alpha\right) \psi^{\prime}+\left(4|\lambda|^{2} v^{2}+p \frac{v^{\prime}}{v}+p(p-\alpha)\right) \psi=0 \\
& \frac{1}{2}\left(\frac{d}{d x}-p+\alpha\right) \phi+\bar{\lambda} v \psi=0 \text { and } \lambda v \phi+\frac{1}{2}\left(\frac{d}{d x}+p\right) \psi=0
\end{aligned}
$$

We fix $p$ in the following lemma.

Lemma 5.4. Let $V \in \mathcal{V}_{4}$. Let $\phi($ resp. $\psi)$ be a solution of $(5.1)\left(\right.$ resp. (5.2)) in $D\left(d^{2} / d x^{2}\right) \cap$ $D\left(v^{2}\right)$. Then the following hold:

(i) If $F_{p} \leq 0$ on $\mathbb{R}$, then $\phi=0$.

(ii) If $F_{\alpha-p} \leq 0$ on $\mathbb{R}$, then $\psi=0$.

Remark. By (5.3), either $\phi=0$ or $\psi=0$ implies that both of them are equal to 0 .

Proof. We prove part (i). Taking the $L^{2}(\mathbb{R})$-inner product of $(5.1)$ with $\phi$ yields that

$$
\left\|\phi^{\prime}\right\|^{2}+\|(2 \lambda v) \phi\|^{2}+\left\langle\left(\frac{v^{\prime}}{v}-\alpha\right) \phi^{\prime}, \phi\right\rangle-\left\langle(p-\alpha)\left(\frac{v^{\prime}}{v}-p\right) \phi, \phi\right\rangle=0 .
$$

Via integration by parts, we have

$$
2 \operatorname{Re}\left\langle\left(\frac{v^{\prime}}{v}-\alpha\right) \phi^{\prime}, \phi\right\rangle=\left\langle\left\{\left(\frac{v^{\prime}}{v}\right)^{2}-\frac{v^{\prime \prime}}{v}\right\} \phi, \phi\right\rangle
$$

(5.4) implies that

$$
2\left\|\phi^{\prime}\right\|^{2}+2\|(2 \lambda v) \phi\|^{2}=\left\langle\left\{\frac{v^{\prime \prime}}{v}-\left(\frac{v^{\prime}}{v}\right)^{2}+2(p-\alpha)\left(\frac{v^{\prime}}{v}-p\right)\right\} \phi, \phi\right\rangle=\left\langle F_{p} \phi, \phi\right\rangle .
$$

By the hypothesis of part (i) the right hand side of (5.5) is non-positive. But the left hand side of (5.5) is non-negative. Hence the both sides of (5.5) are equal to 0 . Hence it follows that $\phi=0$. In the same way we can prove part (ii).

We are now ready to prove Theorem 5.2. 
Proof of Theorem 5.2. By the assumption of this theorem and Lemma 5.4, we immediately obtain the desired result.

A concrete example of superpotential satisfying the assumption in Theorem 5.2 is given by

$$
\partial V(z)=\lambda e^{\beta x} e^{i \alpha y}
$$

with constants $\lambda \in \mathbb{C} \backslash\{0\}$ and $\beta>\alpha / 2$.

Remark 5.5. By a reason similar to that given in Remark 4.12, we can see that for $H_{-}$, there exist infinitely many generalized zero-energy states. 


\section{APPENDIX}

In this appendix, we present some facts on the ordinary differential equation,

$$
y^{\prime \prime}-G y=0 \text { on }[0, \infty)
$$

For the proof of them, we refer to, for example, Sec. 9.2 in Ref.10.

We suppose that the function $G$ is positive, continuous and $x G(x) \notin L^{1}(0, \infty)$. Let $y_{0}$ and $y_{1}$ be the solutions of (A.1) with

$$
y_{1}(0)=1, y_{1}^{\prime}(0)=0, y_{0}(0)=0 \text { and } y_{0}^{\prime}(0)=1
$$

Lemma A.1. $y_{0}(x)>0$ and $y_{1}(x)>0$ on $(0, \infty)$.

Lemma A.2. The limit $\alpha=-\lim _{x \rightarrow \infty} y_{1}(x) / y_{0}(x)$ exists and $y_{+}(x)=y_{1}(x)+\alpha y_{0}(x)$ is the unique solution of (A.1) such that

$$
y_{+}(0)=1 \text { and } y_{+}(x) \rightarrow 0 \text { as } x \rightarrow \infty
$$

Moreover, $y_{+}$is monotone decreasing on $(0, \infty)$ and $y_{+}^{\prime} \in L^{2}(0, \infty)$.

We call $y_{+}$in Lemma A.2 the "normalized subdominant" solution.

Lemma A.3. $\lim _{x \rightarrow \infty} y_{1}(x)=\infty$ and $\lim _{x \rightarrow \infty} y_{0}(x)=\infty$. Hence every solution of (A.1) linearly independent of $y_{+}$tends to infinity as $x \rightarrow \infty$.

The following comparison theorem holds.

Lemma A.4. Let $H$ be a function such that $H \geq G$. Let $Y_{+}$be the normalized subdominant solution of $y^{\prime \prime}-H y=0$ and $Y_{0}$ be the solution of it with $Y_{0}(0)=0$ and $Y_{0}^{\prime}(0)=1$. Then

$$
0<Y_{+} \leq y_{+} \text {and } 0<y_{0} \leq Y_{0} \text { on }(0, \infty)
$$

In particular, if there exist $\gamma$ and $\delta>0$ such that $\delta^{2} \leq G \leq \gamma^{2}$, then the following estimate holds:

$$
e^{-\gamma x} \leq y_{+}(x) \leq e^{-\delta x} \quad \text { on } \quad(0, \infty)
$$

\section{ACKNOWLEDGEMENT}

The author would like to thank Professor Asao Arai for helpful discussions. 


\section{References.}

1. E. Witten, Dynamical breaking of supersymmetry, Nucl. Phys. B 188 (1981), 513.

2. E. Witten, Constraints on supersymmetry breaking, Nucl. Phys. B 202 (1982), 253.

3. A. Jaffe, A. Lesniewski and M. Lewenstein, Ground state structure in supersymmetric quantum mechanics, Ann. Phys. 178 (1987), 313.

4. A. Arai, $O n$ the degeneracy in the ground state of the $N=2$ Wess-Zumino supersymmetric . quantum mechanics, J. Math. Phys. 30 (1989), 2973.

5. A. Arai and O. Ogurisu, Meromorphic $N=2$ Wess-Zumino supersymmetric quantum mechanics, J. Math. Phys. 32 (1991), 2427.

6. A. Arai, Existence of infinitely many zero-energy states in a model of supersymmetric quantum mechanics, J. Math. Phys. 30 (1989), 1164.

7. P. Deift, Applications of a commutation formula, Duck Math. J. 45 (1978), 267.

8. Paul R. Chernoff, Essentially self-adjointness of powers of generators of hyperbolic equations, J. Funct. Anal. 12 (1973), 401.

9. M. Reed and B. Simon, Methods of Modern Mathematical Physics Vol. I, II, IV, Academic, New York, 1979.

10. E. Hille, Lectures on Ordinary Differential Equations, Addison-Wesley Publishing Company. 